\title{
Multi-energy ion implantation from high-intensity laser
}

\author{
Mariapompea Cutroneo, \\ Lorenzo Torrisi, \\ Jiri Ullschmied, \\ Roman Dudzak
}

\begin{abstract}
$\overline{\text { Abstract. The laser-matter interaction using nominal laser intensity above } 10^{15} \mathrm{~W} / \mathrm{cm}^{2} \text { generates in vacuum non- }}$ -equilibrium plasmas accelerating ions at energies from tens $\mathrm{keV}$ up to hundreds MeV. From thin targets, using the TNSA regime, plasma is generated in the forward direction accelerating ions above $1 \mathrm{MeV}$ per charge state and inducing high-ionization states. Generally, the ion energies follow a Boltzmann-like distribution characterized by a cutoff at high energy and by a Coulomb-shift towards high energy increasing the ion charge state. The accelerated ions are emitted with the high directivity, depending on the ion charge state and ion mass, along the normal to the target surface. The ion fluencies depend on the ablated mass by laser, indeed it is low for thin targets. Ions accelerated from plasma can be implanted on different substrates such as Si crystals, glassy-carbon and polymers at different fluences. The ion dose increment of implanted substrates is obtainable with repetitive laser shots and with repetitive plasma emissions. Ion beam analytical methods (IBA), such as Rutherford backscattering spectroscopy (RBS), elastic recoil detection analysis (ERDA) and proton-induced X-ray emission (PIXE) can be employed to analyse the implanted species in the substrates. Such analyses represent 'off-line' methods to extrapolate and to character the plasma ion stream emission as well as to investigate the chemical and physical modifications of the implanted surface. The multi-energy and species ion implantation from plasma, at high fluency, changes the physical and chemical properties of the implanted substrates, in fact, many parameters, such as morphology, hardness, optical and mechanical properties, wetting ability and nanostructure generation may be modified through the thermal-assisted implantation by multi-energy ions from laser-generated plasma.
\end{abstract}

Key words: high-intensity laser $\bullet$ implantation $\bullet$ material modification

\section{Cutroneo}

Nuclear Physics Institute ASCR,

25068 Rez, Czech Republic,

Tel.: +420 266172467

E-mail: cutroneo@ujf.cas.cz

L. Torrisi

Department of Physics Sciences - MIFT,

University of Messina,

V. le F. S. d'Alcontres 31, 98166 S. Agata, Messina, Italy

J. Ullschmied, R. Dudzak

Institute of Plasma Physics ASCR, v.v.i.,

18221 Prague 8, Czech Republic

Received: 17 September 2015

Accepted: 2 November 2015

\section{Introduction}

The dominant regime in most of experiments on ion acceleration from solid targets is the target normal sheath acceleration (TNSA). It is used in laser-matter interaction for laser intensities ranging approximately between $10^{16}$ and $10^{22} \mathrm{~W} / \mathrm{cm}^{2}$ as reported in several publications $[1,2]$. Several models and scaling laws have been suggested to predict the maximum ion energy of accelerated ions in the TNSA regime on the basis of the laser and target parameters [3].

A recent study [4] showed the significant acceleration effect observed at laser intensities of the order of $10^{16} \mathrm{~W} / \mathrm{cm}^{2}$. Experimental detection of ions with energy higher than $4 \mathrm{MeV} /$ charge state as well as the generation of multi-MeV electrons and of intense UV and X-ray emission are referred to the TNSA regime applied to thin foils [4].

A few recent publications, despite reporting the high value of laser irradiance required into the TNSA, support the idea that an increase in laser intensity is generated by decreasing the laser fo- 
cal spot size and at the intensity of interest in this work $\left(10^{16} \mathrm{~W} / \mathrm{cm}^{2}\right)$, it is considered that the TNSA is the dominant regime [5]. It can occur at lower irradiances, that is, of the order of $10^{15-16} \mathrm{~W} / \mathrm{cm}^{2}$ and sub-nanosecond pulse duration producing ion acceleration in the forward direction. The conditions are sufficient to produce hot plasmas and protons of energy of the order of $4 \mathrm{MeV}$ [4]. The beams of emitted ions by laser-matter interaction have a wide range of applications: thin film deposition, ion implantation, nuclear reaction, radiotherapy, etc.

In the present work, we investigate the use of the ion beams to implant substrates placed in the high vacuum chamber. The main advantage is the multi-energetic and multi-species ion implantation, which means that substrate modifications depend on the specific ion energy loss along their trajectories $(\mathrm{d} E / \mathrm{d} x)$, which is directly proportional to the nuclear charge $Z$ of the projectile and depends on the Bragg peak depth position in the substrate matter.

\section{Experimental set-up}

A terawatt iodine laser employed in this experiment is available at the PALS research infrastructure [6] in Prague, Czech Republic. It works at nominal laser irradiance of $10^{15} \mathrm{~W} / \mathrm{cm}^{2}, 1315 \mathrm{~nm}$ of wavelength, 300 ps pulse duration.

Figure 1 shows the sketch of the terawatt iodine laser system set-up. The laser beam enters the interaction chamber with an angle of $30^{\circ}$ with respect to the target normal. It was focused by an aspherical lens $(f=627 \mathrm{~mm}$ for $1 \omega) 29 \mathrm{~cm}$ in diameter onto the target surface, providing a spot of $70 \mu \mathrm{m}$ in diameter. The solid target used to be irradiated in vacuum $\left(10^{-6} \mathrm{mbar}\right)$ were squares $6 \mathrm{~mm} \times 6 \mathrm{~mm}$ of pure $(99.99 \%)$ Au foils $10 \mu \mathrm{m}$ in thickness.

The pulse laser energy ranged between 600 and $560 \mathrm{~J}$ and the focal point between 0 and $-200 \mu \mathrm{m}$ (in front of the target surface), to enhance the ion fluencies on the implanted substrates.

The substrates were mounted at $0^{\circ}, 10^{\circ}$, and $15^{\circ}$ with respect to the target normal and at distance of about $30 \mathrm{~cm}$. The diagnostic system consisted of SiC detectors biased at $-600 \mathrm{~V}$ positioned in front of the target. They were connected to a fast oscilloscope operating at $2 \mathrm{GS} / \mathrm{s}$ to store the recorded spectra.

The incident beam reaches the solid gold target and generates plasma in the forward direction if the $10 \mu \mathrm{m}$ thin $\mathrm{Au}$ is irradiated. The emitted photons,



Fig. 1. Scheme of the experimental set-up. electrons, ions and clusters are deposited onto the substrates placed inside the high-vacuum chamber due to electronic excitation and nuclear collision mechanisms.

The ion range, concentration and depth profiles were carried out by Rutherford backscattering spectrometry (RBS) by means of $2.0 \mathrm{MeV} \mathrm{He}{ }^{+}$ion beam at Tandetron Laboratory, at the Nuclear Physics Institute ASCR, Rez, in the Czech Republic. The scattered ions were detected in Cornell geometry at $170^{\circ}$ then processed by Ultra-Ortec PIPS detector and data evaluation was performed off-line by GISA software [7]. A typical $\mathrm{He}^{+}$ion current for each analysis was about 4.6 nA. Measurements were compared with the theoretical predictions by using SIMRA code [8].

The substrates wettability was evaluated from measuring the static contact angle (SCA) by sessile drop method. The distilled water $(\mathrm{W})$ has been used as a probe liquid. A calibrated $2 \mu \mathrm{l}$ micropipette was used to transfer a small amount of liquid on the substrate surfaces. The measurements were carried out at room temperature $\left(20^{\circ}\right)$. A digital camera captured the pictures for each droplet and the Golden ratio software provides the SCA evaluation as the average of five measures at five different places onto the substrates. The contact angles were measured after the drop deposition at time $t=0 \mathrm{~s}$ and the static contact angles in the equilibrium state at $t=$ $20 \mathrm{~s}$ after the deposition [9]. The measured error for SCA measurements has been $\pm 2^{\circ}$.

\section{Results and discussion}

During the free expansion in vacuum of laser-generated plasma, the emitted ions are accelerated and their current signal was measured by $\mathrm{SiC}$ detectors [10] in time of flight (TOF) configuration. Figure 2a shows the TOF signal for the gold target irradiated at $600 \mathrm{~J}$ pulse energy with a focal position of $-100 \mu \mathrm{m}$.

The ion yield signal amplitude was $142 \mathrm{mV}$ for protons, $127 \mathrm{mV}$ for carbon ions and $157 \mathrm{mV}$ for $\mathrm{Au}$ ions detected in the forward direction energetic protons at $42.5 \mathrm{~ns}$ and the slowest peaks are due to $\mathrm{C}$ at $57.9 \mathrm{~ns}$ and $\mathrm{Au}$ ions at $83.9 \mathrm{~ns}$. The double proton peak was probably due to the two sides of the $\mathrm{Au}$ foil producing proton emission. The faster peak was due to protons emitted from the rear side of the $\mathrm{Au}$ foil, while the slower protons were probably due to the proton emission from the front Au surface and they could lose energy in plasma before they are detected in the forward direction. On the other side, the TOF difference between the two peaks and the yield difference is compatible with the energy loss of protons in the produced plasma and with the proton scattering in plasma. The second slower proton peak really is lower in intensity because the contribution of faster carbon ions should be subtracted to the net peak. However, a correct interpretation of the presence of two proton peaks and of the TOF difference of proton detection is in progress and it is object of actual measurements of accelerated protons coming from interfaces in multi-layered targets. 
a

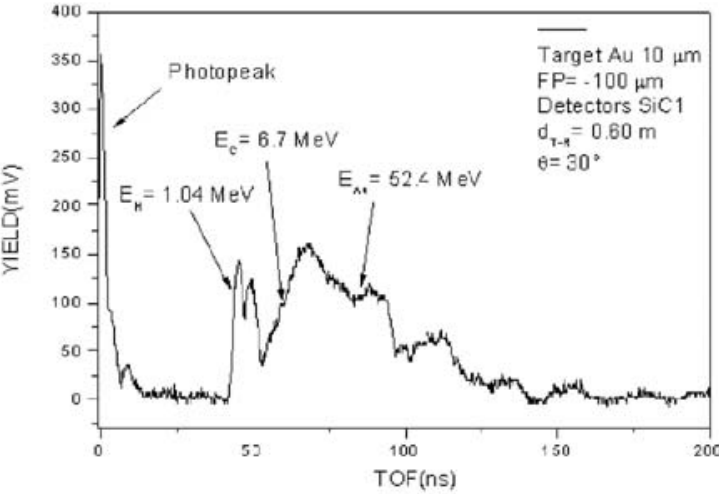

b

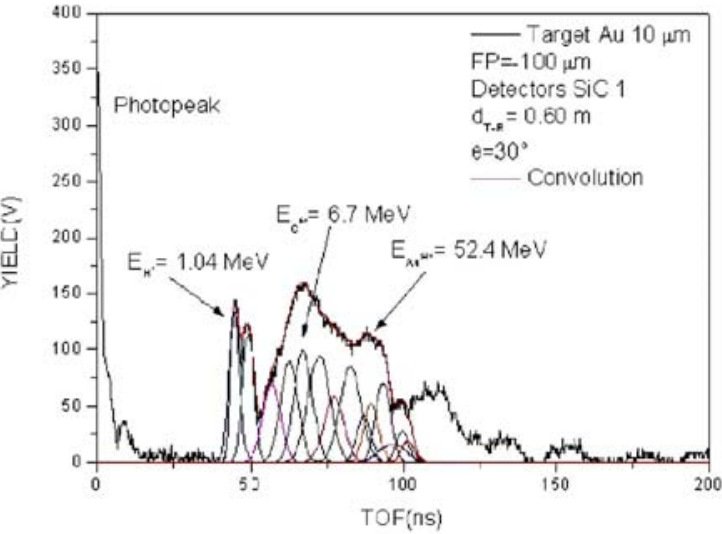

Fig. 2. SiC spectrum of plasma emission in the forward direction, as measured using 10 micron $\mathrm{Au}$ foil (a), and ion deconvolution using the Coulomb-Boltzmann-shifted distributions (b).

Due to the SiC target distance of $60 \mathrm{~cm}$, such TOF values indicate that the maximum kinetic energy is $1.04,6.7$ and $52.4 \mathrm{MeV}$ for protons, carbon and $\mathrm{Au}$ ions, respectively. This result indicates an ion acceleration of about $1 \mathrm{MeV}$ per charge state and also demonstrates that $\mathrm{C}$ ions are full ionized and that the $\mathrm{Au}$ ions have a maximum charge state of about $52+$. Due to the ionization potential of $\mathrm{Au}^{52+}$ of $5.01 \mathrm{keV}$, the expected plasma electron temperature is of the same order of magnitude. Charge states lower than $52+$ are not exhibited in Fig. 2.

The main plasma ion signal contains a convolution of the different ion charge states constituting the plume, from single charge states up to the six charge state for $\mathrm{C}$ ions and the $52+$ charge state for $\mathrm{Au}$ ions. Assuming the distributions to follow a Coulomb-Boltzmann-shifted distribution, it is possible to separate the three ion contributions as reported in Fig. 2b. Of course, the contribution of $\mathrm{C}$ and $\mathrm{Au}$ ions is due to the convolution of six and 52 Boltzmann-like distributions.

Figure 3 shows a typical RBS spectrum relative to the analysis of the Au ion implantation on a $\mathrm{Si}$ substrate placed at $30^{\circ}$ in the forward with respect to the target normal direction and due to 12 laser shots given to the same target of gold in the same TNSA conditions. The spectrum shows the presence a thin surface layer containing $\mathrm{Au}$ atoms from surface to a maximum depth of about 1 micron and the presence of Ge impurities (due to a previous experiment in vacuum chamber irradiating Ge targets). The Au concentration decreases from the surface in

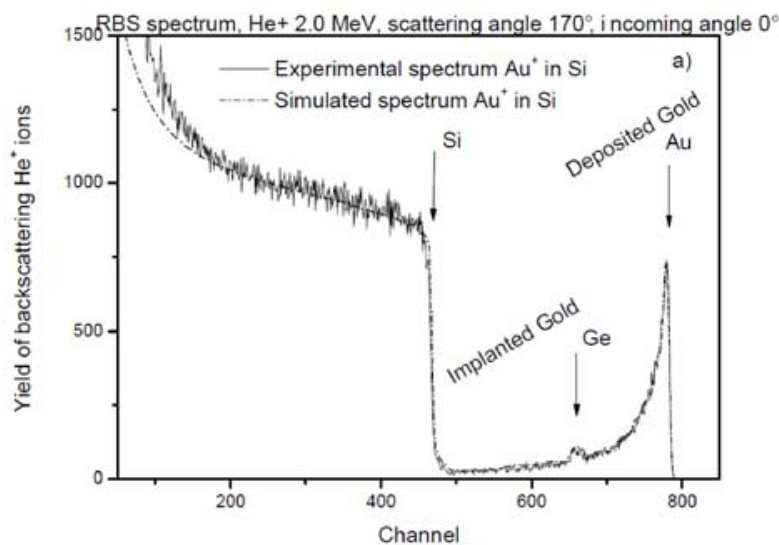

Fig. 3. RBS spectrum (the black solid line) and simulated one (the dot-dashed line) for a Si substrate with laser-implanted Au ions.

the substrate depth. Its maximum concentration, of about $100 \%$, is measured in the first surface layers with a thickness of about $50 \mathrm{~nm}$, indicating that a thin film of gold is deposited due to neutral atoms and very low energetic ions emitted from plasma. RBS [11] shows that the concentration of the Au at $100 \mathrm{~nm}$ depth in silicon is of the order of $40 \mathrm{~atm} \%$; at $200 \mathrm{~nm}$, the value falls to $20 \%$ and at $500 \mathrm{~nm}$, the implantation is below $10 \%$ decreasing at about 0.05 atm $\%$ at about 1 micron depth, confirming the implantation of $\mathrm{Au}$ ion occurred at high energy but at low concentration. The maximum energy of the $\mathrm{Au}$ ions is $4.5 \mathrm{MeV}$; in fact, this beam has a range of 1 micron in silicon, as calculable using SRIM code of Ziegler. The limited energy of implanted ions is due to the Si exposition at $30^{\circ}$ and not at $0^{\circ}$ at which the maximum acceleration occurs.

Figure 4 presents the depth profile of the $\mathrm{Au}$ deposited and implanted in the Si substrate. The integral amount of the implanted Au (total area subtended by the plot of Fig. 5) is $2.08 \times 10^{15}$ atoms $/ \mathrm{cm}^{2}$.

The wettability describes the deposit of a liquid drop on a solid surface. The drop is poured onto the solid surface and a 'like-cap' is formed at the solid-liquid interface when the cohesive forces inside the drop and the adhesive one (due to the gravity forces, the surface tension), which promotes the spreading are in balance. The formed three-phase line at the

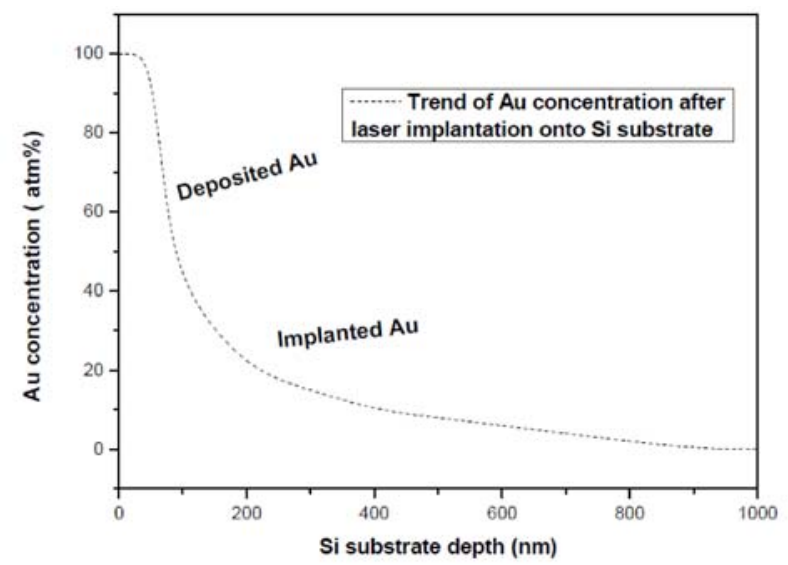

Fig. 4. Au deposited and implanted concentration vs. Si substrate depth. 

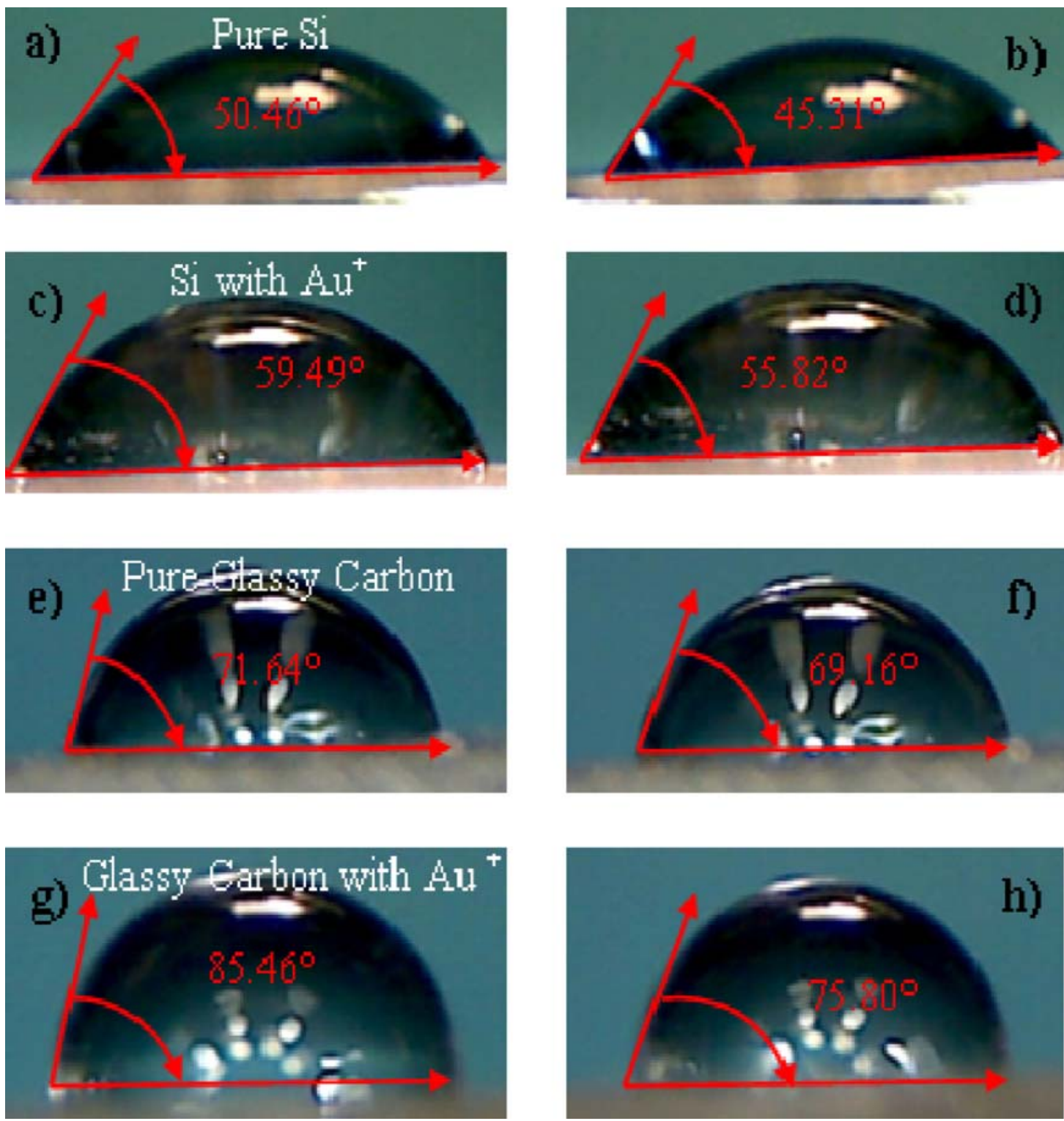

Fig. 5. Images of water drop upon a pure Si substrate at $t=0 \mathrm{~s}(\mathrm{a})$ and at $t=20 \mathrm{~s}(\mathrm{~b})$; Si with implanted Au ions at $t=0 \mathrm{~s} \mathrm{(c)}$ and $t=20 \mathrm{~s} \mathrm{(d)}$; pure glassy carbon at $t=0 \mathrm{~s}(\mathrm{e})$ and $t=20 \mathrm{~s} \mathrm{(f);} \mathrm{glassy} \mathrm{carbon} \mathrm{with} \mathrm{implanted} \mathrm{Au} \mathrm{ions}$ at $t=0 \mathrm{~s}(\mathrm{~g})$ and $t=20 \mathrm{~s}(\mathrm{~h})$.

equilibrium corresponds to the minimal energy state and identifies the contact angle in the meeting point between solid-liquid and solid-vapour interfaces [12]. The Young's equation describing this state is:

$$
\gamma_{s}=\gamma_{s l}+\gamma_{l} \cos \theta
$$

where $\gamma_{s}$ is the surface free energy of a solid, $\gamma_{s l}$ is the surface free energy of solid-liquid interface, $\gamma_{l}$ is the surface free energy of the probe liquid.

Figure 5 presents the $\operatorname{SCA}(\theta)$ of different liquids on Si-pristine and on $\mathrm{Si}$ with $\mathrm{Au}$ implanted from laser-generated plasma. The contact angle increases going from Si-pristine to Si-implanted and to other substrates such as glassy carbon-pristine and glassy carbon $\mathrm{Au}$ implanted. The contact angle is also reported just as deposited the drop (left) and after $20 \mathrm{~s}$ from the deposit (right). Further surface analyses were performed in order to have information on the modification induced by the plasma ion implantation. Surface analysis such as scanning electron microscopy (SEM), atomic force microscopy (AFM), X-ray fluorescence (XRF) and X-ray diffraction (XRD) have demonstrated that after the laser implantation, metallic $\mathrm{Au}-\mathrm{Si}$ nanometric structures are developed onto the substrate surfaces that make the treated $\mathrm{Si}+\mathrm{Au}$ substrates more hydrophilic and the wettability increases compared to the virgin Si surface.

\section{Conclusions}

In order to make the laser-driven ion beams appropriate for applications such as medical research area [13], implantation, microelectronics and radiography, the ion beam parameters need to be reproducible. Many groups are turning their efforts in this direction.

Presently, the implantation of mono-crystal Si has been performed using pico-second high-intensity laser in TNSA regime. The emitted multispecies 
present energies with Boltzmann distributions ranging between some $\mathrm{keV}$ up to tens of $\mathrm{MeV}$. The implanted ions modify the composition and the morphology of the surface affecting the bulk properties and under the high plasma temperature may produce new compounds and nanostructured species on the implanted substrate.

The SCA measurements performed using different probe liquids, with a known surface energy, are used to estimate the changing occurring in the surface layers under plasma laser-generated implantation. The increase in the contact angles was attributed to the increase of the surface enhancements due to the presence of metallic ions and clusters.

Acknowledgments. The research has been realized at PALS lab and CANAM (Center of Accelerators and Nuclear Analytical Methods) infrastructure LM 2011019 and has been supported by the Research Collaboration Agreement between NPI and IPP of ASCR and PPL of the University of Messina and project no. P10 8/12/G108, respectively.

This work was performed at NPI ASCR, Rez, Czech Republic, the University of Messina, Italy, and the Institute of Plasma Physics ASCR, Prague, Czech Republic.

\section{References}

1. Borghesi, M. (2013). Laser-driven ion acceleration: State of the art and emerging mechanisms. Nucl. Instrum. Methods Phys. Res. Sect. A-Accel. Spectrom. Dect. Assoc. Equip., 740, 6-9. DOI: 10.1016/j. nima.2013.11.098.

2. Macchi, A., Borghesi, M., \& Passoni, M. (2013). Ion acceleration by superintense laser-plasma interaction. Rev. Modern Phys., 85(2), 751-793. DOI: 10.1103/ RevModPhys.85.751.

3. Lecz, Z., Boine-Frankenheim, O., \& Kornilov, V. (2013). Target normal sheath acceleration for arbitrary proton layer thickness. Nucl. Instrum. Methods Phys. Res. Sect. A-Accel. Spectrom. Dect. Assoc. Equip., 727, 51-58. DOI: 10.1016/j.nima.2013.05.163.

4. Torrisi, L., Cutroneo, M., Calcagno, L., Rosinski, M., \& Ullschmied, J. (2014). TNSA ion acceleration at $10^{16} \mathrm{~W} / \mathrm{cm}^{2}$ sub-nanosecond laser intensity. $J$. Phys. Conf. Ser., 508, 012002. DOI: 10.1088/17426596/508/1/012002.

5. Brenner, C. M., Green, J. S., Robinson, A. P. L., Carroll, D. C., Dromey, B., Foster, P. S., Kar, S., Li, J. T., Markey, K., Spindloe, C., Streeter, M. J. V., Tolley, M., Wahlström, C. G., Xu, M. H., Zepf, M., McKenna, P., \& Neely, D. (2011). Dependence of laser accelerated protons on laser energy following the interaction of defocused, intense laser pulses with ultra-thin targets. Laser Part. Beams, 29, 345-451. DOI: 10.1017/ S0263034611000395.

6. Torrisi, L., Giuffrida, L., Cutroneo, M., Cirrone, P. G. A., Picciotto, A., \& Krasa, J. (2012). Proton emission from thin hydrogenated targets irradiated by laser pulses at $10^{16} \mathrm{~W} / \mathrm{cm}^{2}$. Rev. Sci. Instrum., 83, $02 \mathrm{~B} 315$. DOI: 10.1063/1.3673506.

7. Saarilahti, J., \& Rauhala, E. (1994). Interactive personal-computer data analysis of ion backscattering spectra. Nucl. Instrum. Methods Phys. Res. Sect. B-Beam Interact. Mater. Atoms, 64(1/4), 734-738.

8. Mayer, M. (1999). SIMNRA [computer software]. Max-Planck-Institut fur Plasmaphysik. Web site actual 2014: www.rzg.mpg.de/ mam/.

9. Kubiak, K. J., Wilson, M. C. T., Mathia, T. G., \& Carras, S. (2011). Dynamics of contact line motion during the wetting of rough surfaces and correlation with topographical surface parameters. Scanning, 33, 370-377. DOI: 10.1002/sca.20289.

10. Cutroneo, M., Musumeci, P., Zimbone, M., Torrisi, L., La Via, F., Margarone, D., Velyhan, A., Ullschmied, J., \& Calcagno, L. (2013). High performance SiC detectors for $\mathrm{MeV}$ ion beams generated by intense pulsed laser plasmas. J. Mater. Res., 28, 87-93. DOI: 10.1557/jmr.2012.211.

11. Cutroneo, M., Mackova, A., Malinsky, P., Matousek, J., Torrisi, L., \& Ullschmied, J. (2015). High-intensity laser for Ta and Ag implantation into different substrates for plasma diagnostics. Nucl. Instrum. Methods Phys. Res. Sect. B-Beam Interact. Mater. Atoms, 354, 56-59. DOI: 10.1016/j.nimb.2014.11.082.

12. Scolaro, C., Torrisi, L., Cutroneo, M., \& Velardi, L. (2014). Wetting ability modifications in biocompatible polymers induced by pulsed lasers. $J$. Phys. Conf. Ser., 508, 012030. DOI: 10.1088/17426596/508/1/012030.

13. Torrisi, L. (2015). Gold nanoparticles enhancing protontherapy efficiency. Recent Patents on Nanotechnology, 9(1), 1000-000. DOI: 10.2174/187221012798109255. 